\title{
PRINCIPLES OF EXERCISE THERAPY
}

BY

\author{
ERNEST A. NICOLL, M.D., B.Ch., F.R.C.S.Ed. \\ Surgical Director, Accident Service, Mansfield General Hospital; Surgeon-in-Charge, Miners' Rehabilitation Centre. \\ Berry Hill Hall
}

Remedial exercises have long been recognized as the basis of functional recovery in fractures and other injuries of the locomotor system. In fact, the success of any accident service depends very largely on its organization for the application of therapeutic exercise to all cases and at all stages. In nearly all prolonged or serious illness, however, both medical and surgical, and non-traumatic as well as traumatic, there is a general deterioration of the locomotor system, and this is the chief cause of incapacity during the convalescent stage. In the past, convalescence from these conditions has been entirely negative and loosely supervised, but efforts are now being made by the Ministry of Health to substitute a more positive approach on the lines of the after-care organization in accident services. This, it is hoped, will be brought about by the more extended use of therapeutic exercise, on both a prophylactic and a curative basis. It should be a relatively easy proposition if the right type of organization can be evolved, because it is well known that deterioration of the locomotor system through simple disuse or toxaemia is much easier to cure than the same deterioration following trauma.

Two conditions, however, are essential to success. First, organization is necessary. The benefits of exercise therapy must be brought to all cases at the right time, in the right dosage, and in the appropriate form. Secondly, the physicians and surgeons themselves must appreciate and understand the basic principles of exercise therapy; for only then can they be expected to direct its application and to supervise intelligently the work of their technical assistants - the physiotherapist, the occupational therapist, and the gymnast. It is a defect in medical education that there is practically no teaching in this important branch of therapy, and even our most up-to-date textbooks do little more than advise us to use it. The object of this paper is to formulate such basic principles, for this is all that is required in order to direct the work of technical assistants. The busy specialist cannot be expected to acquire a detailed knowledge of remedial exercises, occupational therapy, gymnastics, recreational therapy, and the like ; nor is this necessary. The experience out of which this paper is compiled was gained in directing the work of an accident service-and particularly the work of the Miners' Rehabilitation Centre at Berry Hill Hall - so it has a particular bearing on fractures and other injuries of the locomotor system. But principles are principles, and should remain true for any extension beyond this field. It is hoped, therefore, that, while being of particular interest to those engaged in the traumatic surgery of the locomotor system, the general principles here discussed will also interest that much larger body of physicians and surgeons who are now being urged by the Ministry of Health to incorporate exercise therapy into their systems of treatment. The general principles to be discussed may be stated as follows :
Therapeutic exercise must be : (1) both specific (focal) and general; (2) administered with due regard to dosage ; (3) rhythmic in regard to contraction and relaxation; (4) progressive in $(a)$ range, $(b)$ power, and $(c)$ time; $(5)$ variable in form-the chief forms being (a) medical gymnastics, (b) occupational therapy, and $(c)$ recreational therapy.

It is beyond the scope of this paper to discuss the detailed application of these principles. Where this is done it is merely in order to exemplify the principle.

\section{Focal and General Exercises}

Specific (or focal) exercises are those which pick out some particular muscle group for selective redevelopment. This in effect means devising exercises which make the desired muscle group work as a prime mover. It may be asked why this is necessary-why, for example, cannot these muscles be developed by general activity? The answer is that in a good many cases they can, but that in such cases the result depends on any general movement in which the muscle normally takes part being performed in the normal manner. In fact, however, most patients learn more quickly how to dodge the use of inefficient or painful muscles. In other words, they learn trick movements resulting in compromises. But compromises are not desirable if the normal mechanism can be regained, so focal exercises differ from general exercises in stressing, not the end to be attained, which is functional movement, but the means whereby it is attained, which is the production of the movement by a particular muscle group acting as a prime mover.

The use of focal exercises, therefore, is of most importance in injuries of the locomotor system, for in these conditions certain muscles and joints are affected a great deal while the rest are comparatively normal. Such a situation lends itself much more readily to the development of trick movements than does a generally weakened locomotor system following ordinary sickness ; for if the whole system is depressed the muscles still retain their normal relative action even if each muscle is itself inefficient. Trick movements, on the other hand, depend on functional inequality. All faulty gaits, for example, are trick movements acquired by a patient who is made to walk when his muscles or joints are physically incapable of producing a normal gait, and it is the duty of the surgeon to pick out the particular muscles and joints concerned and redevelop them by focal exercises in order to prevent a temporary compromise from developing into a fixed habit. From these arguments it is obvious that general exercises alone, properly supervised, will usually be sufficient for the deterioration following ordinary sickness, but that in injuries affecting specific parts of the locomotor system a combination of both general and focal exercise is necessary.

An example of the need for deliberate "focusing" in exercise technique is afforded by the quadriceps. This important muscle group is wrongly regarded as a single muscle. In fact, it behaves more like two separate muscles, the vastus internus 
being quite selective in its action. Extension of the knee-joint to within about 15 degrees of fiii movement can be brought about entirely by the rectus femoris, vastus lateralis, and vastus intermedius, under conditions of minimal stress. It is only when the stress is markedly increased, as in single-leg weightbearing, that the vastus internus enters into this range. That is why a weak vastus internus can be "caught out" in such activities as walking up and down stairs, running, and alternateleg skipping. On the other hand, the last 15 degrees of extension, even against minimal stresses, cannot be effected without the vastus internus. This latter range is so small compared with the rest, however, that a patient may appear to be extending and flexing his knee-joint most energetically and using his quadriceps most effectively, yet never really using his vastus internus at all. This explains why "quadriceps drill" in plaster casts flexed more than 15 degrees at the knee-joint results in selective wasting of the vastus internus. This excessive flexion is quite unnecessary to prevent rotation of the fragments, and all long plasters should fix the knee well within the range of the vastus internus. It also explains why quadriceps drill on a flexed Thomas splint results in the same condition. Where this is necessary, as in supracondylar and lowerthird fractures of the femur, exercises should be supplemented by selective faradism and the position of the knee brought to within 15 degrees of full extension as soon as possible. If any surgeon doubts this he should have himself put up in a flexed plaster or on a flexed splint and then try to do quadriceps drill and see if he can use the vastus internus-even when it is normal. The importance of all this is that the vastus internus is the key muscle in producing the last 15 degrees of extension and stabilizing the joint in all positions. If it is inefficient a syndrome is created familiar to all orthopaedic surgeons-the joint that " gives way " under stress or sudden unexpected movement. Of the two components of the muscle, the rectus femoris group can most safely be left to take care of itself because no compensatory mechanism can be devised in its absence-but the vastus internus needs the most focal and specific treatment because it operates over such a small range that a compensatory mechanism can be evolved. Once evolved it may become a fixed habit, and general activity will confirm rather than cure it. Further wasting then occurs, leading to the paradox of progressive disuse atrophy in an active patient. So the term "quadriceps drill" should be abolished and "vastus internus drill" substituted.

It is beyond the scope of this paper to go into questions of how to evolve special exercises for the redevelopment of vastus internus or other particular muscle groups-the object is merely to underline the principle that focal exercise technique is essential in certain conditions and to give the reasoning on which this opinion is based. It is necessary at the present time to make this point for two reasons-first, because it is being represented in certain quarters that general exercises will cure everything; and, secondly, because of the revival of occupational therapy. In this form of exercise therapy focal technique has not received the attention it deserves, and is apt to be disregarded entirely or even condemned. This will be referred to later.

The need for general exercises as a part of any system is self-evident. Here the body as a whole is exercised, and the injured muscles learn to act again as fixators, synergists, and antagonists as well as prime movers. Muscles were never intended by Nature to be individualists, so exercises aimed at their selective rédevelopmant must always be combined with exercises aimed at co-ordinating them with the rest of the locomotor system-a process often referred to as "re-education."

\section{The Dosage Principle}

Exercise therapy has been used in the past in such small and scattered doses that it may truly be said to have been on a homoeopathic basis. Having been "discovered," however, the new danger is that it will be given in poisonous doses. It is necessary, therefore, to point out that weak muscles can be poisoned by overdosage with exercise. Two factors have to be considered in this connexion-first, the optimum stress under which a muscle should exercise ; and, secondly, the relative duration of exercise periods and rest periods. Generally speaking, the weaker the muscle the less should be the demands made on it, both in stress and in time. At Berry Hill Hall weak muscles are never exercised against more than $25 \%$ of their "all out" efficiency, but this proportion is increased to $50 \%$ as the muscle strengthens. At the same time the exercise periods are lengthened and the rest periods shortened.

\section{The Principle of Rhythm}

All therapeutic exercises should be rhythmic. Contraction must be followed by relaxation, and the relaxation must be complete and long enough to allow normal circulatory conditions to be restored in the muscle before it begins its next contraction. A good instructor teaching his patients will be heard uttering over and over again the incantation: "Contract ... and rest . . . contract . . . and rest." Mr. J. H. C. Colson, former superintendent at Berry Hill Hall, introduced this principle into resisted pulley-and-weight exercises by incorporating ingenious "relaxation stops" in the pulley circuits (Fig. 2). He also insisted, most rightly, on its observance in occupational therapy. For this reason carpentry-which is mostly a matter of sustained contraction-was never used to any great extent in finger cases, whereas the craft-work known as netting, which involves a regularly alternating contraction and relaxation, was used extensively. There are other reasons why carpentry, the most vaunted of all forms of occupational therapy in official quarters, is also the worst. What is the scientific basis underlying this principle ? In a muscle affected by disuse or injury the circulatory and lymphatic systems are impaired. Atrophy, even in non-traumatic cases, is always associated with intramural fibrosis. In a traumatized muscle there are many additional factors producing fibrosis, and fibrosis in muscle always impairs the circulatory condition by causing obstruction to venous and lymphatic drainage. Now, muscle more than any other tissue depends on good circulatory conditions, because the products of contraction (chiefly sarcolactic acid), unless speedily carried away, act as a muscle poison, and fatigue results. So in an atrophied or fibrotic muscle it is essential to give the embarrassed circulation time to clear the breakdown products-and hence exercises should be rhythmic in character as opposed to exercises which call for sustained contractions.

\section{The Principle of Progression}

The principle of progression is one of the most important and firmly established features of the Ling system of gymnastics. But its use in remedial exercises, in which it is much more necessary, has been neglected-and the same applies even more so to occupational therapy. It is not sufficient that a muscle should be made to contract-it must contract against gradually increasing stresses, through gradually increasing ranges of movement, and over gradually increasing periods of time. It is not a matter of devising an exercise to make a muscle work; the task of the physiotherapist is to devise a whole series of exercises which provide smooth progression from the stage of extreme weakness to the stage of full use against the stresses of normal work. This progression must be in three dimensions-in range of movement, in power of movement, and in duration under stress-but by far the greatest of these is power. Range without power is worse than useless, for in its extreme form we have a flail-joint. Power without range, on the other hand, is compatible with full function in heavy industry. So progression in power is of absolutely vital importance in exercise therapy.

But to consider first the methods of increasing range of movement. Passive movements, at one time widely practised, are now almost universally condemned. Active mosements, on the other hand, are universally recommended. But what fundamental differences are there that place them such poles apart? Surely only two factors are involved: first, that the force applied is much greater in passive movement ; and, secondly, that this greater force is not under the patient's control. Now there is no reason why the force available should not be increased-in fact, this is desirable. Suppose, for example, that the patient is trying to mobilize a stiff shoulder limited in abduction, and the normal muscles available to produce this movement are wasted and weakened-as they are likely to be. Would any surgeon object if the power of those muscles were suddenly increased to normal or even a little above 
normal? On the contrary, he would welcome it. So the only objection to passive movement must be that this desirable extra force is provided by an outside agency-and that is, of course, a very legitimate objection. But suppose that by means of a pulley circuit (Fig. 1) the abductors of one arm

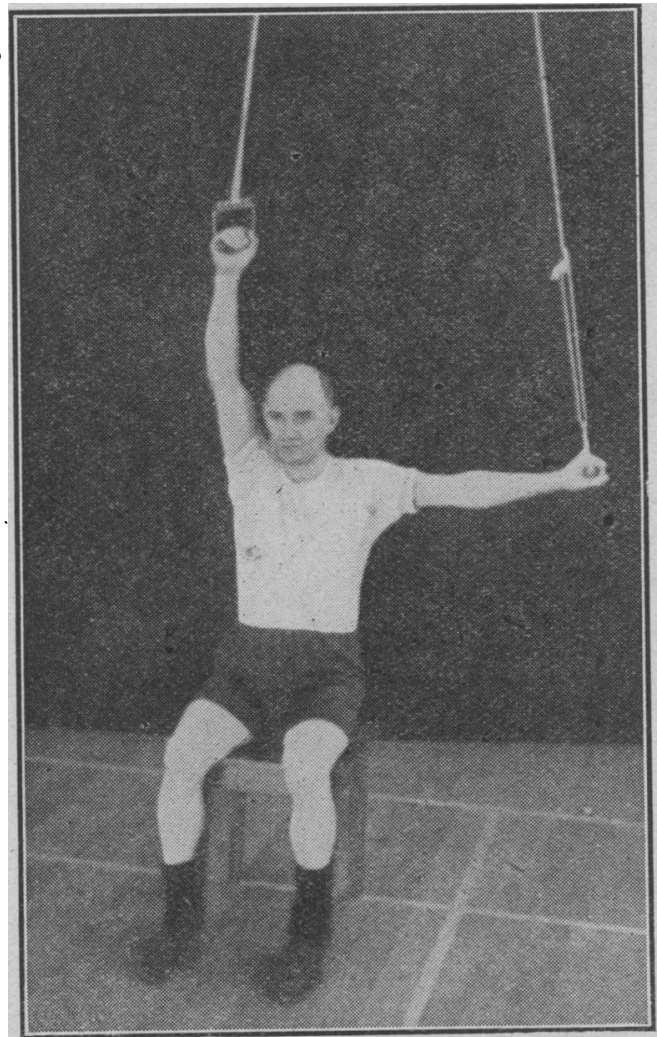

FIG. 1.-Remedial exercise with a pulley circuit for self-assisted active movement.

are reinforced by the adductors of the opposite arm-then the extra force available for breaking down adhesions and stretching contracted tissues is still under the patient's control. This type of exercise has been developed at Berry Hill Hall for practically all the important joints of the body, and has been called "auto-assisted active movement (A.A.A.)." Even the proximal interphalangeal joints of the fingers can be mobilized in this way by strapping the stiff finger above and below the joint to its fellows on either side. The following is an example of the use of the pulley circuit.

The patient shown in Fig. 1-a miner aged 50-sustained a severe shoulder injury which resulted in complete stiffness. At the beginning of treatment at Berry Hill Hall he had no shoulder movement at all, and was so weak he couldn't lift a bucket. It took three months' hard work to cure this most difficult case; but it was worth every minute of it, for he has regained full movement and full power, and is now back at his job as a coal-face miner.

Apart from its use in recovering movement, this type of exercise has another indication, and that is to preserve movement that would otherwise be lost through painful inhibition. To take the shoulder once more as an example-it is well known that many stiff painful shoulders result from simple contusion, and the difference in prognosis between a dislocation due to a fall on the hand without damage to the deltoid and one associated with contusion to the deltoid is equally well known. Contusion in certain muscles, of which the deltoid is one, produces severe pain when the muscle contracts under stress. To guard against this the patient elevates his arm by fixing the shoulder-joint and rotating the scapula, thus producing the familiar "reversed rhythm" seen in these cases. Painful inhibition of movement in a contused muscle leads to fibrosis ; fibrosis leads to more pain, and so the vicious circle goes on till it ends as a "frozen shoulder." What was originally a painful inhibition becomes a painful contracture. There are two aims of treatment in the early stages of such a condition -to cure the pain and so release the inhibition, and to preserve the normal range of movement during this process, which may be prolonged. The best way to preserve movement in these cases is by the use of auto-assisted active exercises. Another method is by using a weight to assist the movement. This can be done on the same pulley apparatus, the weight being gradually diminished as improvement allows. Once the stress is removed the muscle is able to contract without pain, and normal rhythm and full range are restored. Strictly speaking, this is not an example of progression in range-it is a special use of exercise technique to prevent loss of range; but it embodies some very important general principles.

Progression in power is usually obtained in gymnastics by making the performer do the exercise from different gymnastic positions. In the simplest form of the exercise a position and a technique are adopted which allow the performer to obtain help from accessory muscles. In the various progressions these are cut out by degrees. Another gymnastic method of obtaining progression in power is by using the lever principle in reverse. For instance, in extension of the spine from the prone position, the arms are first kept at the sides, then clasped behind the neck, and finally fully stretched out ahead of the patient. A third method is to start by performing the exercise in "inner range" and gradually progress to "outer range" movement on the theory that all muscles have to act more powerfully in outer range.

But the most scientific way of obtaining progression in power is to add a resistance which can be accurately measured. This is achieved very simply and very effectively by the use of pulley-and-weight apparatus, the principle of which is illustrated in Fig. 2. This type of apparatus is used only for the focal

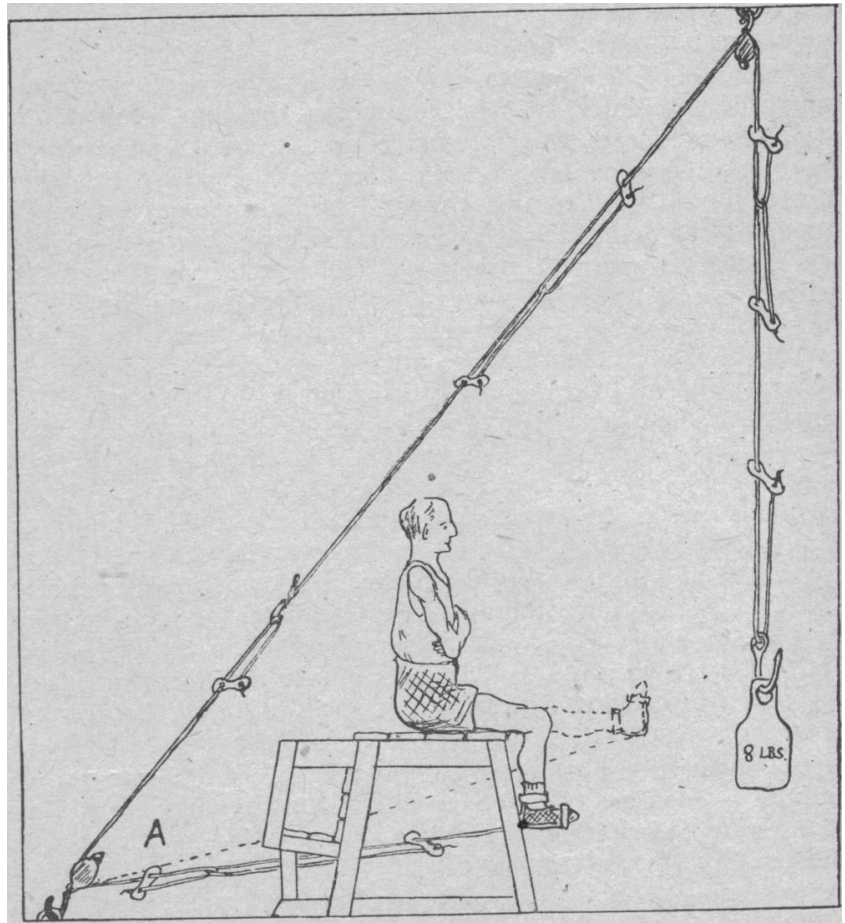

Fig. 2.-The principle of the calibrated resistance exercise $(\mathrm{A}=$ relaxation stop)

type of exercise, so it is essential to isolate the muscle effectively as a prime mover. The "relaxation stop," mentioned earlier, is a knot or metal runner in the cord arranged in such a way that it engages in the pulley and takes the entire strain of the weight in the resting position of the exercise.

It is extremely important in using this type of exercise to pay due regard to the dosage principle. Failure to do this has produced chronic sprains, especially in knee cases, and has led several surgeons to issue warnings against the use of resisted exercises altogether for the knee. This is quite wrong, because the principle of progression in power is unchallengeable, and the apparatus evolved to achieve it is beyond criticism. The fault lies in the use of the method without due regard to the dosage and relaxation principles. At Berry Hill Hall no ill effects have been observed when using the technique 
described earlier in discussing dosage. Another way to produce sprains in knee cases is by failing to redevelop the vastus internus and then making it work against the same resistance as the rectus femoris component. This in its turn is due to failure to recognize the vastus internus as a separate muscle and therefore to use the entirely separate pulley circuit appropriate to its action.

The third type of progression in exercise therapy is progression in time-that is, carrying out the exercise under the same stress for increasing periods. This is very important in rehabilitating industrial workers, because industry demands not only that a workman shall be able to exercise his muscles against certain stresses but that he shall be able to do so continuously for whatever number of hours constitutes a "shift."

\section{Principle of Variation in Form}

If exercise therapy is to be carried out for many hours a day it must be disguised in various forms to make it palatable to the patient. Medical gymnastics alone would become boring; the patient would lose interest and initiative, and therefore cease to put his best endeavours behind them. So exercise therapy must be capable of assuming different forms, though in doing so it must conform as nearly as possible to the general principles already laid down. Unfortunately this is not always the case, and in the two chief disguises-occupational therapy and recreational therapy-the art of "sugarcoating" the pill has proved so diverting that the ingredients of the pill itself have been rather overlooked. Another reason why there must be variation in form is that medical gymnastics do not lend themselves to certain conditions. For example, no patient would sit in a gymnasium flexing and extending his fingers for six hours a day, but he will do it in an occupationaltherapy department.

It is beyond the scope of this paper to discuss either occupational therapy or recreational therapy, but there are a few points in regard to both that bear on principles. Occupational therapy is used, of course, in two quite different ways - as a mental diversion (with which this paper is not concerned) and as a disguised form of remedial exercise. Employed in the latter sense, it becomes more important than ever to ensure that the proper muscles and joints are used, and that the patient is not simply learning trick movements. This can often be achieved by fixing the compensatory joints in plaster-e.g., in netting by fixing the metacarpo-phalangeal joints in order to make the patient use his proximal interphalangeal joints. Forms of craftwork should be favoured in which the movements are an alternate contraction and relaxation, such as weaving; those involving impaction stresses and sustained contraction should be avoided. Progression in range and power should not be lost sight of, and with ingenuity both can be incorporated into most forms of occupational therapy. For example, looms have been designed at Berry Hill Hall for shoulder, forearm, wrist, knee, and ankle movements, each with its own mechanism for ensuring effective muscle isolation and progression in both range and power.

The chief aim of the surgeon in all this should be not to acquire a detailed knowledge of occupational therapy but to satisfy himself that the essential principles of therapeutic exercise are being satisfied. Very little need be said about recreational therapy. It is a form of general exercise in which some sort of rough progression is possible, as, for example, in progressing from simple sitting games to walking and then running games. Games can never be very specific, nor is it desirable that they should be ; their chief role is to redevelop. general bodily function in a psychologically stimulating atmosphere, and the principal concern of the surgeon will be to protect his patients against the possible ill effects of their own enthusiasm or that of their fellows.

\section{Summary}

The basic principles of exercise therapy have been discussed and the following five requirements laid down:

(1) Exercises must be both focal and general. (2) They must be administered with due regard to correct dosage. This is variable from case to case and at different stages in the same case. (3) They must be rhythmic in character. (4) They must be progressive in range, power, and time. (5) They must be variable in form, the chief forms being gymnastic, occupational, and recreational.

It is considered that a knowledge of these principles is all that is required or can be expected of the surgeon or physician who has to incorporate exercise therapy into his system of treatment. The detailed application and elaboration of principles is the province of the technical assistants in the various forms of exercise therapy.

\section{JAUNDICE FOLLOWING ADMINISTRATION OF HUMAN BLOOD PRODUCTS BY}

H. V. MORGAN, M.B., M.R.C.P.

Physician, E.M.S.; Chief Assistant to the Medical Professorial Unit, St. Bartholomew's Hospital AND

D. A. J. WILLIAMSON, M.B., B.S. Surgeon Lieutenant, R.N.V.R.; Late Chief Assistant, St. Bartholomew's Hospital

At the present time human blood and its derivatives are being widely used for transfusion purposes. It therefore seems relevant to describe a small series of cases of jaundice which have followed the intravenous administration of plasma or reconstituted dried human serum.

\section{Summary of Literature}

Findlay and MacCallum (1937, 1938; with Murgatroyd, 1939) reported the occurrence of 89 cases clinically resembling infective hepatitis among 3,100 persons inoculated against yellow fever from 36 days to 7 months previously. The vaccine consisted of yellow fever virus suspended in human serum, which was obtained at first from patients who had recovered from yellow fever, but latterly from healthy donors without a past history of jaundice. The volume of serum injected in each case never exceeded $0.275 \mathrm{c} . \mathrm{cm}$. These observers clearly demonstrated that the disease was not a form of yellow fever, and suggested that it was due to a hepatotoxic virus in the serum. Soper and Smith (1938) and Fox et at. (1942) recorded similar cases in South America.

A widespread outbreak has recently occurred among members of the American Forces inoculated against yellow fever (Memorandum on Yellow Fever Jaundice, 1942). 28,585 cases were reported during the first half of 1942, 62 of which were fatal. The clinical condition, which again resembled " catarrhal jaundice" except for the occurrence of joint pains and urticaria in $20 \%$ of cases, developed 40 to 120 days after inoculation. Necropsy showed acute or subacute necrosis of the liver, sometimes associated with nodular hyperplasia. Of particular interest is the complete absence of contact cases.

MacNalty (1937) reported 37 cases of acute infective jaundice, with seven deaths from acute hepatic necrosis, occurring 16 to 100 days after the administration of convalescent measles serum. All the 37 cases were from a group of 82 to 109 persons inoculated with serum of a particular batch. Propert (1938) in describing seven of these cases of infective hepatitis mentioned the occurrence of jaundice in two uninoculated children who two months previously had been in contact with some of the cases. Findlay and MacCallum quote several observers who have reported cases of hepatitis occurring in horses after inoculation against a number of equine diseases. The only common constituent of the inocula was horse serum (Theiler, 1919 ; Gordon, 1935 ; Marsh, 1.937 ; Slagsvold, 1938).

\section{Clinical Material}

Late in May, 1942, an atypical case of jaundice occurred at an E.M.S. hospital (Case 3). A fortnight later a patient discharged from this same hospital (Case 4) was admitted to an associated hospital with a similar clinical condition. Comparison of the two cases showed that both had received intravenous serum several weeks previously. It was therefore felt that these might be examples of the type of jaundice caused by the administration of serum. An investigation of all cases known to have ${ }^{3}$ transfused with serum or plasma in the past year begun, and the following results were obtained. 